\title{
Longitudinal buckling of slender pressurised tubes
}

\author{
S. Syngellakis \\ Wessex Institute of Technology, UK
}

\begin{abstract}
This paper is concerned with Euler buckling of long slender tubes subjected to internal pressure. A small disturbance from its perfectly straight form is imposed to the tube to account for the effect of the non-conservative, follower forces generated by fluid pressure. An equivalent lateral distributed force is derived by considering the geometric changes arising from the flexural deformation of the tube. A new differential equation governing the buckling mode of the tube considered as a column is obtained. This equation includes the inertia term for investigating buckling through the dynamic stability criterion. The theory is applied to several cases of pressurised tubes under various loadings and support conditions. The results show that internal fluid pressure can have a significant load transfer role that may lead to buckling in certain cases but has no apparent effect in others.

Keywords: cylindrical tubes, internal fluid pressure, Euler buckling.
\end{abstract}

\section{Introduction}

Axially compressed cylinders buckle as shells for a wide range of their length to diameter ratio. Due to the engineering relevance of this problem, it has attracted considerable attention and, as a consequence, various issues relating to its analysis have been addressed in a large number of publications. Among the issues that have been investigated is the effect of internal or external fluid pressure on the local shell buckling mode and the respective critical load of such structures or containers [1-4].

Very long slender cylinders may however buckle as elastic columns whose critical load can be computed from the well known Euler's formula. Such columns, usually referred to as cylindrical tubes, may also be subjected to 
internal pressure whose direction is deformation dependent and therefore nonconservative. An engineering design comprising axially compressed and internally pressurised tubes is shown in fig. 1 . This is a schematic representation of a hydraulic ram consisting of two internally, fluid-pressurised tubes within a co-axial outer cylinder. The outer tube slides over the inner one and lifts the weight $P$ under the action of the liquid pressure $p_{f}$ and the gas pressure $p_{g}$ existing within the volume between the inner tube and the cylinder. The former generates a force $P_{o 2}$ acting on the top end plate of the outer tube, the latter a supporting force $P_{g}$ at its base. The inner tube is directly subjected to a compressive axial force $P_{i 2}$ also due to the liquid pressure $p_{f}$.

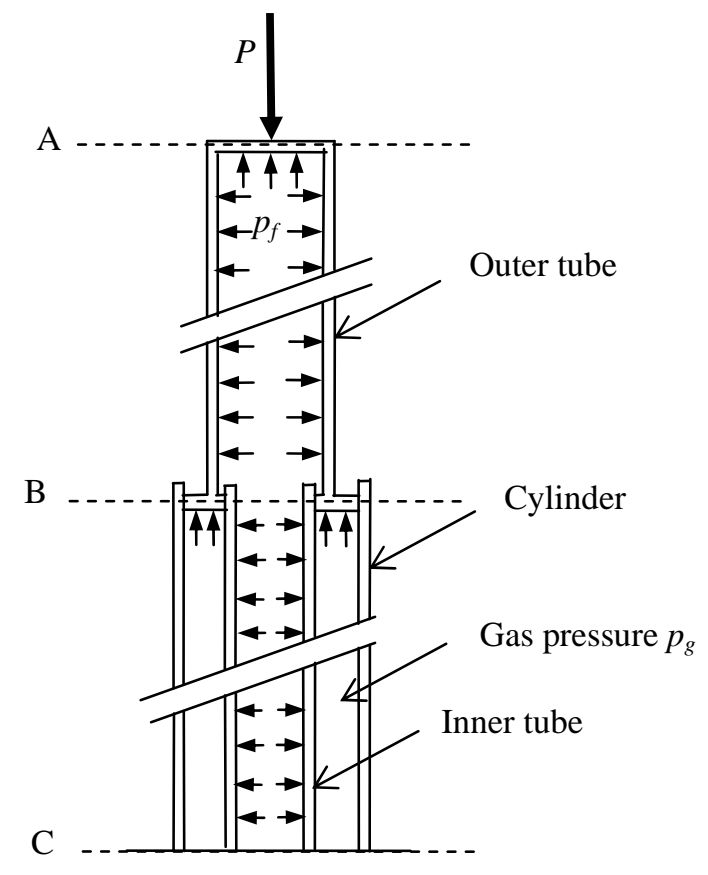

Figure 1: $\quad$ Schematic of hydraulic ram design.

Given the material and geometric characteristics of both tubes, their Euler buckling loads can be determined and compared to the respective axial loads. For the outer tube in particular, the internal pressure reduces the applied compressive force by $P_{o 2}$ thus appears to increase, effectively, the critical load by the same amount. The Euler solution however is based on the assumption that a slightly buckled form does not affect the orientation of the applied forces. This is not true for any of the three forces $P_{o 2}, P_{i 2}$ or $P_{g}$, which remain tangent to the deformed axis of the tube after buckling. It is also necessary to examine whether the pressure on the flexurally deformed wall of the tubes contributes to their instability. 
The aim of the present analysis is to assess the influence of internal pressure on the stability of a long open or closed tube. Since fluid pressure produces nonconservative, follower forces, this problem is addressed by applying a small initial disturbance from the perfectly straight, vertical position. If the disturbance is static, the problem is formulated by considering the so called adjacent equilibrium of the tube. This approach was adopted by Kounadis [5] who investigated a number of non-classical buckling problems involving long tubes under axial compression and internal pressure. The effectiveness and reliability of the adjacent equilibrium approach to such problems is re-examined here by attempting to model more rigorously the interaction between the pressurised fluid and the flexurally deformed tube wall. It is shown that, for cantilevered tubes, the determination of the critical load may not be always possible by this method. In certain such cases, the applicability and effectiveness of the dynamic criterion of stability is discussed. For tubes pinned at both ends however, buckling is predicted for critical loads obtained using the Euler formula.

\section{Bending of pressurised tubes}

Referring to the deformed tube element shown in fig. 2, the extension of a longitudinal fibre $\delta s$ at a distance $y$ from the neutral axis is

$$
\Delta(\delta s)=-\frac{y}{R} \delta s
$$

where $R$ is the radius of curvature of the deformed beam given by

$$
\frac{1}{R}=\left[1+\left(\frac{\mathrm{d} v}{\mathrm{~d} x}\right)^{2}\right]^{-\frac{3}{2}} \frac{\mathrm{d}^{2} v}{\mathrm{~d} x^{2}} \cong \frac{\mathrm{d}^{2} v}{\mathrm{~d} x^{2}},
$$

$v(x)$ the deflection and the approximation is valid for very small deflection. Thus, the longitudinal strain in the tube should be given by

$$
\varepsilon_{\chi}=-\frac{y}{R}
$$

Applying Hooke's law, the stress is given by

$$
\sigma_{x}=E \varepsilon_{x}=-E \frac{y}{R}
$$

and the bending moment by

$$
M(x)=-\int_{A} y \sigma_{x} \mathrm{~d} A=\frac{E I}{R} \cong E I \frac{\mathrm{d}^{2} v}{\mathrm{~d} x^{2}}
$$

In a straight tube with inner diameter $D$, subjected to internal pressure $p$, two opposite forces, both equal to $p D(\delta s)$, are acting on the inner wall surfaces of the element above and below the neutral axis. In a bent tube, the surface under 


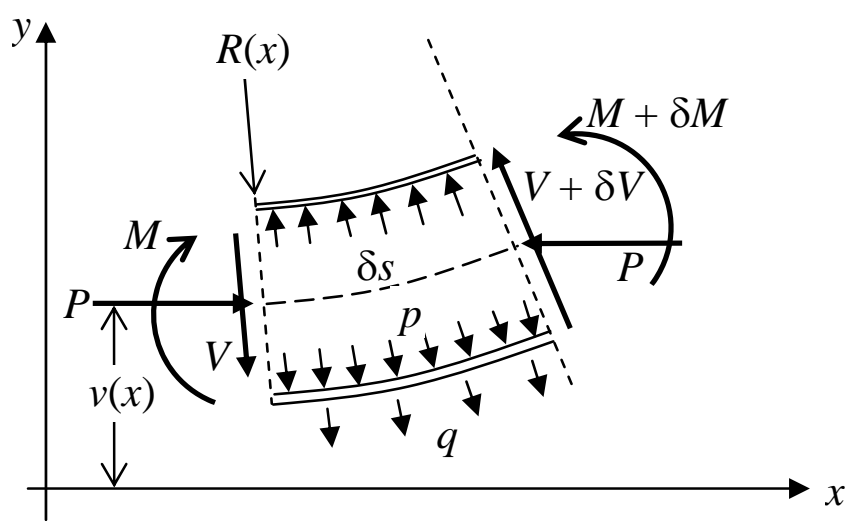

Figure 2: $\quad$ Deformed configuration of a beam element of length $\delta s$.

tension is greater than that under compression. Thus, for $y>0$, the pressure applied on a projected area is reduced by

$$
\int_{-D / 2}^{D / 2}[\Delta(\delta s)] \mathrm{d} z=(\delta s) \int_{-D / 2}^{D / 2} \frac{y}{R} \mathrm{~d} z=\frac{D^{2}(\delta s)}{4 R} \int_{-\pi / 2}^{\pi / 2} \cos ^{2} \beta \mathrm{d} \beta=\frac{\pi D^{2}(\delta s)}{8 R}
$$

where the cross-sectional angular co-ordinate $\beta$ is defined in fig. 3 and $y=$ $(D / 2) \cos \beta, z=(D / 2) \sin \beta$.

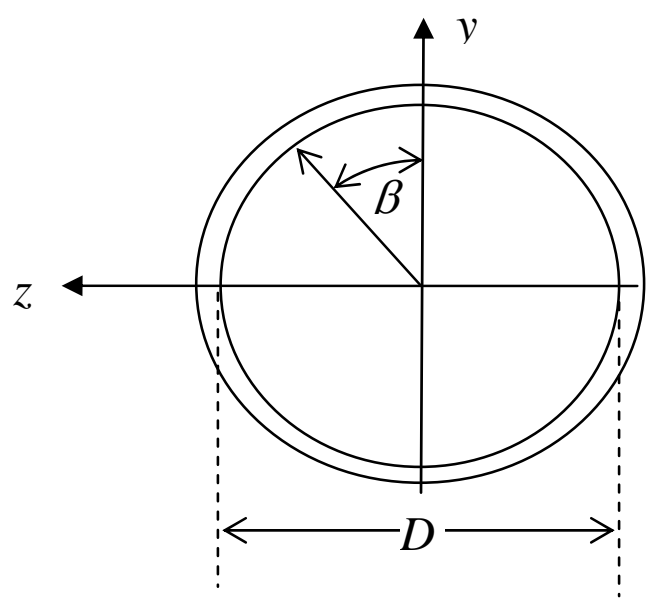

Figure 3: $\quad$ Cross section of a hollow tube.

For $y<0$, the projected area is increased by exactly the same amount, so that bending generates a lateral force per unit length of the deformed beam axis, 
normal to the axis in the direction from the compressed to the extended fibres of the beam. According to eqn (3), this force is given by

$$
q=p \frac{\pi D^{2}}{4 R} \cong P_{f} \frac{\mathrm{d}^{2} v}{\mathrm{~d} x^{2}}
$$

where $P_{f}$ is the force acting on the end plate of a closed tube or on the piston imposing the pressure in an open tube.

Equilibrium of forces acting on the tube element of fig. 2, in the direction normal to the axis of the tube, gives

$$
-V+\left(V+\frac{\partial V}{\partial x} \mathrm{~d} x\right)-q \mathrm{~d} x=m \frac{\partial^{2} v}{\partial t^{2}} \mathrm{~d} x
$$

where $V$ represents the shear force, $m$ the mass per unit length and $t$ the time. The right hand side accounts for small lateral oscillations which are imposed when the dynamic criterion of stability is applied. Moment equilibrium of the same tube element gives

$$
-M+\left(M+\frac{\partial M}{\partial x} \mathrm{~d} x\right)+V \mathrm{~d} x+P \frac{\partial v}{\partial x} \mathrm{~d} x=0
$$

where $P$ is the net axial force applied in the undeformed $x$ direction. Eqns (5) and (6) are simplified to

$$
\begin{gathered}
\frac{\partial V}{\partial x}-q=m \frac{\partial^{2} v}{\partial t^{2}} \\
\frac{\partial M}{\partial x}+V+P \frac{\partial v}{\partial x}=0
\end{gathered}
$$

Substituting $V$ from eqn (8) into eqn (7) and accounting for eqns (2) and (4) finally gives

$$
\frac{\partial^{2}}{\partial x^{2}}\left(E I \frac{\partial^{2} v}{\partial x^{2}}\right)+\frac{\partial}{\partial x}\left(P \frac{\partial v}{\partial x}\right)+P_{f} \frac{\partial^{2} v}{\partial x^{2}}+m \frac{\partial^{2} v}{\partial t^{2}}=0
$$

The solution of the above equation is sought under various applied axial loads $P$ and boundary conditions.

\section{Tubular cantilevers}

The analysis is first applied to open vertical tubes, fixed at their base and free at the top, subjected to two types of internal pressure. In the first problem, the tube is subjected to the fluid's and its own weight. The tube is filled with fluid with specific weight $\gamma_{f}$ so that the fluid pressure at height $x$ is $\gamma_{f}(L-x)$. Accounting for the weight of the tube wall itself generates a vertical compressive force $P=\gamma_{t} A_{t}(L-x)$, where $\gamma_{t}$ is the specific weight of the tube material and $A_{t}$ the cross section of the tube.

Considering the static equilibrium of the tube at a slightly deformed configuration as shown in fig. 4, eqn (9) becomes 


$$
E I \frac{\mathrm{d}^{4} v}{\mathrm{~d} x^{4}}+\left(\gamma_{t} A_{t}+\gamma_{f} A_{f}\right)(L-x) \frac{\mathrm{d}^{2} v}{\mathrm{~d} x^{2}}-\gamma_{t} A_{t} \frac{\mathrm{d} v}{\mathrm{~d} x}=0
$$

For an empty tube under its own weight, the governing equation can be directly obtained from eqn (8) by setting $V=0$. The solution to this problem is given by Timoshenko and Gere in their classical monograph [6]. In the case of a tube filled with a liquid, the problem represented by eqn (10) is certainly more complex than that formulated by Kounadis [5], who simply added the weight of the liquid to that of the solid tube and thus obtained essentially the same solution as that presented by Timoshenko and Gere.

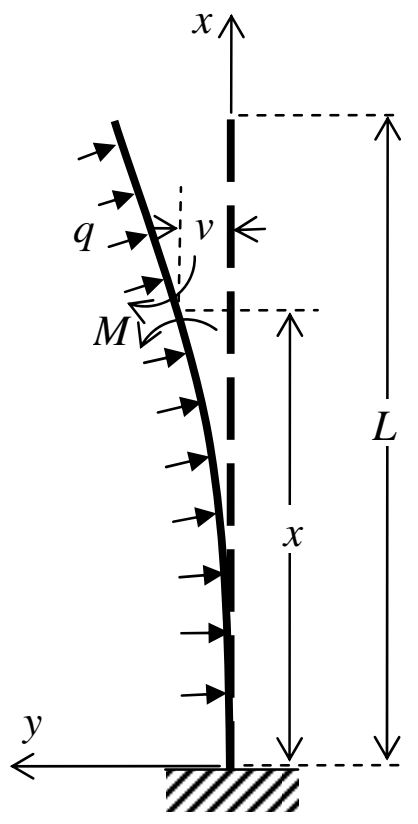

Figure 4: Cantilever tubes under internal pressure.

Eqn (10) can be simplified if the total weight per unit mass of the filled tube is assumed to be much larger than that of the tube wall alone. Then, the third term on the left hand side of eqn (10) can be neglected and the substitution

$$
u=\frac{\mathrm{d} v}{\mathrm{~d} x}
$$

leads to the equation

$$
E I \frac{\mathrm{d}^{3} u}{\mathrm{~d} x^{3}}+\left(\gamma_{t} A_{t}+\gamma_{f} A_{f}\right)(L-x) \frac{\mathrm{d} u}{\mathrm{~d} x}=0
$$

This is identical to that governing the deflection of an empty tube under its own weight and a general solution for $u$ can be found in terms of Bessel 
functions in exactly the same manner [6]. The boundary conditions that should be satisfied by $u$ are however different. More specifically,

$$
\frac{\mathrm{d}^{2} u}{\mathrm{~d} x^{2}}=\frac{\mathrm{d} u}{\mathrm{~d} x}=0 \text { at } x=L
$$

since both shear force and bending vanish there. It is possible to show that, for the solution to satisfy both conditions (13), the constants of integration must both vanish. Therefore, only the straight form of equilibrium is possible. This conclusion is consistent with that reached in the case of a concentrated follower force applied at the top of a column [6]. It may be possible to determine the critical load using the dynamic criterion of stability but this is not attempted here because of the mathematical complexity of the problem and the apparent lack of engineering interest in its solution.

The tube can also be subjected to a high uniform pressure $p_{f}$ relative to which the pressure due to the fluid's weight can be neglected. In such a case, the adjacent equilibrium of the tube is governed by

$$
\frac{\mathrm{d}^{2}}{\mathrm{~d} x^{2}}\left(E I \frac{\mathrm{d}^{2} v}{\mathrm{~d} x^{2}}\right)+P_{f} \frac{\mathrm{d}^{2} v}{\mathrm{~d} x^{2}}=0
$$

in which $P_{f}$ is constant. It has been shown by Timoshenko and Gere that this equation leads to the straight tube as the only possible equilibrium configuration. Subsequent application of the dynamic criterion of stability [6] provided a formula for the critical value of $P_{f}$ from which the critical pressure can be obtained using eqn (4). A complete account of this solution was more recently presented by Kounadis [5].

\section{Hydraulic ram}

The fully extended ram can be assumed as firmly held in place against any lateral movement at the three levels A, B and C shown in fig 0 . The two ends of each tube may also be subject to a degree of rotational constraint, but this is expected to be far from the ideal clamped end condition. The tubes are therefore analysed with the conservative assumption of pinned support at both their ends.

\subsection{Outer tube}

The free body diagram of the top, outer tube at a slightly deformed configuration is shown in fig. 5(a). At the two ends of the tube, internal pressure generates the forces

$$
P_{o 2}=p_{f} \frac{\pi D_{o i}^{2}}{4}=P_{o f}, P_{o 1}=p_{f} \frac{\pi\left(D_{o i}^{2}-D_{i o}^{2}\right)}{4}
$$

where $D_{o i}$ and $D_{i o}$ are, respectively, the inner diameter of the outer tube and the outer diameter of the inner tube.

Thus, the static version of eqn (9) becomes in this case, 


$$
\frac{\mathrm{d}^{2}}{\mathrm{~d} x^{2}}\left(E I_{o} \frac{\mathrm{d}^{2} v}{\mathrm{~d} x^{2}}\right)+\frac{\mathrm{d}}{\mathrm{d} x}\left[\left(P-P_{o f} \cos \theta_{2}\right) \frac{\mathrm{d} v}{\mathrm{~d} x}\right]+P_{o f} \frac{\mathrm{d}^{2} v}{\mathrm{~d} x^{2}}=0
$$

where $E$ is the Young's modulus of the material, $I_{o}$ is the second moment of cross-sectional area, while $P_{\text {of }}$ is obtained from eqn (4) for $p=p_{f}$ and $D=D_{o i}$. For very small deviations from the straight line configuration, $\cos \theta_{2} \cong 1$ and eqn (16) is reduced to

$$
E I_{o} \frac{\mathrm{d}^{4} v}{\mathrm{~d} x^{4}}+P \frac{\mathrm{d}^{2} v}{\mathrm{~d} x^{2}}=0
$$

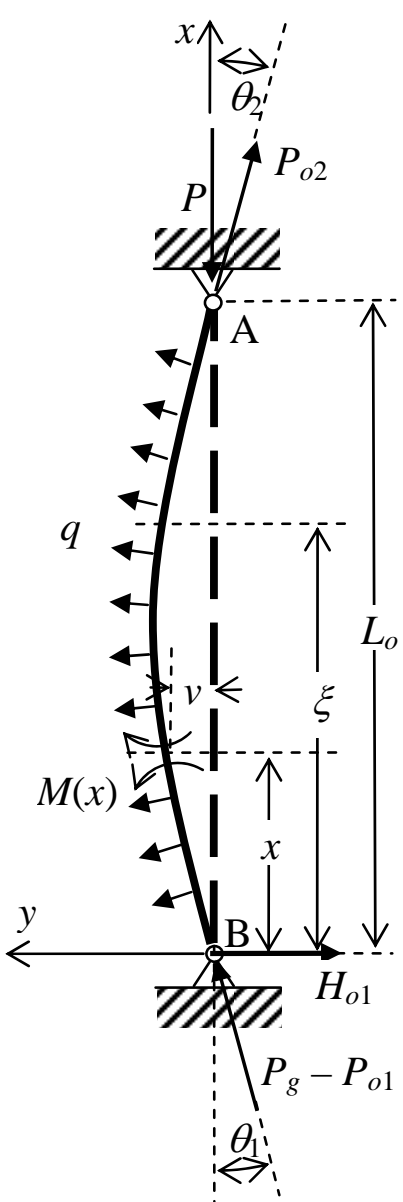

(a)

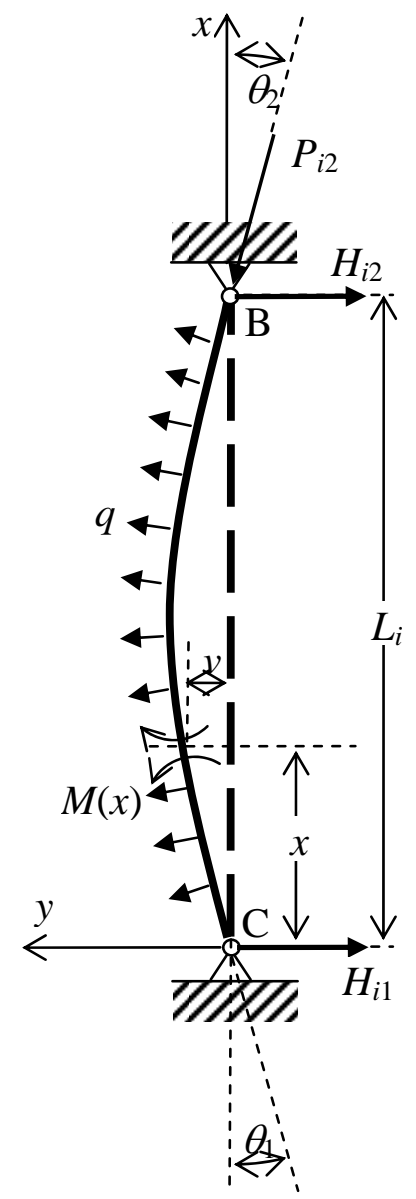

(b)

Figure 5: $\quad$ Buckled profiles of the tubes; (a) outer tube, (b) inner tube. 
But this is the differential equation governing the buckling mode of a straight column subjected to a conservative compressive force $P$. The critical value of $P$ for the assumed pinned-pinned end conditions is thus provided by the well known Euler formula

$$
P_{c}=\frac{\pi^{2} E I_{o}}{L_{o}^{2}}
$$

where $L_{o}$ the length of the outer tube. It is thus evident that internal pressure has no effect on the buckling capacity of the outer tube.

An alternative form of the equation of equilibrium can be deduced by obtaining the expression for $M(x)$ and substituting it in eqn (2). Referring to fig. 5(a), the bending moment at $x$, due to $q$ over $\delta s$ at $\xi(x<\xi)$, is

$$
\begin{gathered}
\mathrm{d} M_{q}=-(\xi-x)\left(\frac{P_{o f}}{R(\xi)} \cos \theta\right) \mathrm{d} s-[v(\xi)-v(x)]\left(\frac{P_{o f}}{R(\xi)} \sin \theta\right) \mathrm{d} s \\
=-(\xi-x) P_{f} \mathrm{~d}(\sin \theta)+[v(\xi)-v(x)] P_{f} \mathrm{~d}(\cos \theta)
\end{gathered}
$$

since $\mathrm{d} s / R=\mathrm{d} \theta$. Integrating by parts over the length $\left(x, L_{o}\right)$ gives

$$
M_{q}(x)=\left(L_{o}-x\right) P_{o f} \sin \theta_{2}-v(x) P_{o f} \cos \theta_{2}
$$

since $\theta\left(L_{o}\right)=-\theta_{2}$ and $v\left(L_{o}\right)=0$. It can be shown that overall equilibrium of the slightly bent column is satisfied for horizontal reactions $H_{o 1}=P \sin \theta_{1}$ at $\mathrm{B}$ and $H_{o 2}=0$ at A. Thus, the total bending moment at $x$ is

$$
M(x)=-\left(L_{o}-x\right) P_{o 2} \sin \theta_{2}-v(x)\left(P-P_{o 2} \cos \theta_{2}\right)+M_{q}(x)=-v(x) P
$$

since $P_{o 2}=P_{o f}$. Therefore, only the conservative force $P$ contributes a moment appearing in the equation governing the deflection, which becomes

$$
E I_{o} \frac{\mathrm{d}^{2} v}{\mathrm{~d} x^{2}}+P v=0
$$

leading to the same classical Euler buckling formula mentioned above.

\subsection{Inner tube}

The free body diagram of the inner tube at a slightly deformed configuration is shown in fig. 5(b). At its top end, the internal pressure generates the compressive follower force

$$
P_{i 2}=p_{f} \frac{\pi\left(D_{i o}^{2}-D_{i i}^{2}\right)}{4}
$$

where $D_{i i}$ is the inner diameter of the inner tube. Hence, the version of eqn (9) governing the static adjacent equilibrium state is, in this case, written

$$
\frac{\mathrm{d}^{2}}{\mathrm{~d} x^{2}}\left(E I_{i} \frac{\mathrm{d}^{2} v}{\mathrm{~d} x^{2}}\right)+\frac{\mathrm{d}}{\mathrm{d} x}\left[P_{i 2} \cos \theta_{1} \frac{\mathrm{d} v}{\mathrm{~d} x}\right]+P_{i f} \frac{\mathrm{d}^{2} v}{\mathrm{~d} x^{2}}=0
$$


where $I_{i}$ is the second moment of cross-sectional area of the inner tube while $P_{\text {if }}$ is obtained from eqn (4) for $p=p_{f}-p_{g}$ and $D=D_{i i}$. For very small deviations from the straight line configuration, $\cos \theta_{2} \cong 1$ and eqn (16) is reduced to

$$
E I_{i} \frac{\mathrm{d}^{4} v}{\mathrm{~d} x^{4}}+P_{e f f} \frac{\mathrm{d}^{2} v}{\mathrm{~d} x^{2}}=0
$$

where $P_{\text {eff }}=P_{i 2}+P_{i f}$. But this is the differential equation governing the buckling mode of a straight column subjected to a follower compressive force $P_{\text {eff. }}$. It can be shown that the critical value of $P_{\text {eff }}$ for the assumed pinned-pinned end conditions is given by

$$
\left(P_{e f f}\right)_{c}=\frac{\pi^{2} E I_{i}}{L_{i}^{2}}
$$

that is, the well known Euler formula where $L_{i}$ is the length of the inner tube. It should be noted that the same critical load is predicted by the dynamic criterion of stability for this particular combination of support conditions.

During normal lift operation, the inner tube is subjected to internal and external pressures of almost identical magnitude; this keeps $P_{\text {eff }}$ at a value lower than that of $P$ and the inner tube is safe against buckling if the outer tube has been designed to that effect. The situation becomes critical in the case of sudden loss of gas pressure. Then the outer tube is assumed fully extended and the oil pressure suddenly increased to a value balancing the total applied load, that is,

$$
p_{f}=\frac{4 P}{\pi D_{i o}^{2}}
$$

As previously argued, this change in internal pressure has no effect on the buckling behaviour of the outer tube whose safety factor remains the same. The safety factor of the inner tube however changes considerably and can be obtained by comparing the applied pressure given by eqn (22) with the critical pressure obtained from

$$
\left(P_{\text {eff }}\right)_{c}=\left(p_{f}\right)_{c} \frac{\pi D_{i o}^{2}}{4}=\frac{\pi^{2} E I_{i}}{L_{i}^{2}}
$$

It is interesting to note by comparing eqn (22) with eqn (23) that $P_{\text {eff }}=P$, that is, the fluid transmits the total conservative applied force from the top of the ram to the inner tube as an axial follower force.

Consideration of overall equilibrium of the slightly bent inner tube provides the horizontal reactions $H_{i 1}=P_{i f} \sin \theta_{1}$ at $\mathrm{C}$ and $H_{i 2}=\left(P_{i 2}+P_{i f}\right) \sin \theta_{2}$ at $\mathrm{B}$. Considering again the end conditions $\theta\left(L_{i}\right)=-\theta_{2}$ and $v\left(L_{i}\right)=0$, the bending moment is deduced as

$$
M(x)=\left(L_{i}-x\right) P_{i 2} \sin \theta_{2}-v(x) P_{i 2} \cos \theta_{2}-\left(P_{i 2}+P_{i f}\right)\left(L_{i}-x\right) \sin \theta_{2}+M_{q}(x)
$$

where $M_{q}(x)=\left(L_{i}-x\right) P_{i f} \sin \theta_{2}-v(x) P_{i f} \cos \theta_{2}$. Hence

$$
M(x)=-v(x)\left(P_{i 2}+P_{i f}\right) \cos \theta_{2} \cong-v(x) P_{\text {eff }}
$$


Substituting the above expression into eqn (2) generates a differential equation yielding the same critical load as that given by eqn (20).

\section{Concluding remarks}

It is surprising that very little work on the Euler buckling of long, slender, internally pressurised tubes is reported in the current technical literature. The problem might have been addressed in early structural studies, access to which is not easy today. The need for designing the hydraulic ram against buckling prompted the present investigation, which led to the derivation of a simple analytical tool based on beam theory for the prediction of the critical load of pressurised tubes using either a static or a dynamic stability criterion.

The analysis shows that buckling predictions depends strongly on the specific loading and support conditions. The non-conservative nature of the fluid loading appears to have a stronger effect on cantilevered columns (fully fixed at the base, free at the top) for which the application of the dynamic stability criterion is necessary for obtaining the critical loads. In contrast, predictions for columns pinned at both ends can be obtained considering the static adjacent equilibrium of a slightly bent form. For the two specific cases of engineering interest analysed, buckling in the first is due only to the applied conservative force while in the other, the follower force acts as an equivalent conservative force. It is interesting to compare these conclusions with the established view that internal pressure has generally a beneficial effect on the buckling capacity of cylindrical shells subjected to axial compression [7].

The highest priority for further work should be the validation of the simple analytical model. This can be experimental and numerical; finite element modelling can be used for the latter but this can be very challenging considering the amount of resources required for a non-linear analysis with adequate mesh refinement of a geometrically simple solid but with strongly disproportional in its dimensions. Once validated, the simple analysis can be further tested through its application to a wider range of loading and support conditions of certain engineering interest and significance.

\section{References}

[1] Greiner, R. and Guggenberger, W., Buckling behaviour of axially loaded steel cylinders on local supports - with and without internal pressure. ThinWalled Structures, 31(1-3), pp. 159-167, 1998.

[2] Tafreshi, A., Buckling and post-buckling analysis of composite cylindrical shells with cutouts subjected to internal pressure and axial compression loads. International Journal of Pressure Vessels and Piping, 79(5), pp. 351359, 2002.

[3] Kyriakides, S. and Paquette, J.A., Plastic buckling of tubes under axial compression and internal pressure. International Journal of Mechanical Sciences, 48(8), pp. 855-867, 2006. 
[4] Hassler, M. and Schweizerhof, K., On the influence of fluid-structureinteraction on the stability of thin-walled shell structures. International Journal of Structural Stability and Dynamics, 7(2), pp. 313-335, 2007.

[5] Kounadis, A.N., Nonclassical stability problems: Instability of slender tubes under pressure. Journal of Aerospace Engineering, 14(1), pp. 6-11, 2001.

[6] Timoshenko, S. and Gere, J.M., Theory of Elastic Stability, McGraw-Hill: New York, 1961.

[7] Engineering Sciences Data Unit, Elastic local buckling stresses of thinwalled unstiffened circular cylinders under combined axial compression and internal pressure, 2006. 